\title{
VIII International Workshop on Computer Graphics and Geometric Modeling - CGGM'2009
}

\author{
Andrés Iglesias \\ Department of Applied Mathematics and Computational Sciences, \\ University of Cantabria, Avda. de los Castros, s/n, E-39005, Santander, Spain \\ iglesias@unican.es \\ http://personales.unican.es/iglesias/
}

\begin{abstract}
This short paper is intended to give our readers a brief insight about the Eight International Workshop on Computer Graphics and Geometric Modeling-CGGM'2009, held in Baton Rouge, Louisiana (USA), May 25-27 2009 as a part of the ICCS'2009 general conference.
\end{abstract}

\section{CGGM Workshops}

\subsection{Aims and Scope}

Computer Graphics (CG) and Geometric Modeling have become two of the most important and challenging areas of Computer Science. The CGGM workshops seek for high-quality papers describing original research results in those fields. Topics of the workshop include (but not limited to): geometric modeling, solid modeling, CAD/CAM, physically-based modeling, surface reconstruction, geometric processing and CAGD, volume visualization, virtual avatars, computer animation, CG in Art, Education, Engineering, Entertainment and Medicine, rendering techniques, multimedia, non photo-realistic rendering, virtual and augmented reality, virtual environments, illumination models, texture models, CG and Internet (VRML, Java, X3D, etc.), artificial intelligence for CG, CG software and hardware, CG applications, CG education and new directions in CG.

\subsection{CGGM Workshops History}

The history of the CGGM workshops dates back nine years ago, when some researchers decided to organize a series of international conferences on all aspects of computational science. The first edition of this annual conference was held in San Francisco in 2001 under the name of International Conference on Computational Science, ICCS. This year ICCS conference is held in Baton Rouge, Louisiana (USA). 
After ICCS'2001, I realized that no special event devoted to either computer graphics or geometric modeling had been organized at that conference. Aiming to fill this gap, I proposed a special session on these topics to ICCS'2002 organizers. Their enthusiastic reply encouraged me to organize the first edition of this workshop, CGGM'2002. A total of 81 papers from 21 countries were submitted to the workshop, with 35 high-quality papers finally accepted and published by Springer-Verlag, in its Lectures Notes in Computer Science series, vol. 2330. This great success and the positive feedback of authors and participants motivated that CGGM became an annual event on its own. Subsequent editions were held as follows (see [1] for details): CGGM'2003 in Montreal (Canada), CGGM'2004 in Krakow (Poland), CGGM'2005 in Atlanta (USA), CGGM'2006 in Reading (UK), CGGM'2007 in Beijing (China) and CGGM'2008 again in Krakow (Poland). All of them were published by Springer-Verlag, in its Lecture Notes in Computer Science series, volumes 2668, 3039, 3515, 3992, 4488 and 5102 with a total of 52, 24, 22, 22, 20 and 16 contributions, respectively. In addition, one Special issue has been published in 2004 in the Future Generation Computer Systems - FGCS journal [2]. Another special issue on CGGM'2007 is available online and in the way to be published in printed form soon.

\section{$2 \quad$ CGGM'2009}

This year CGGM has received a total of 11 papers of which 6 have been accepted as full papers. The reader is referred to [3] for more information about the workshop. The workshop chair would like to thank the authors, including those whose papers were not accepted, for their contributions. I also thank the referees (see the CGGM'2009 International Program Committee and CGGM'2009 International Reviewer Board in [3]) for their hard work in reviewing the papers and making constructive comments and suggestions, which have substantially contributed to improving the workshop.

\section{Acknowledgments}

This workshop has been supported by the Spanish Ministry of Education and Science, National Program of Computer Science, Project Ref. TIN2006-13615 and the University of Cantabria. The CGGM'2009 chair also thanks Dick van Albada, workshops chair of general conference ICCS'2009 for his all-time availability and diligent work during all stages of the workshop organization. Thanks for your support, Dick!

\section{References}

1. Previous CGGM: http://personales.unican.es/iglesias/CGGM2009/Previous.htm

2. Iglesias, A. (Guest ed.): Special issue on "Computer Graphics and Geometric Modeling. Future Generation Computer Systems 20(8), 1235-1387 (2004)

3. CGGM 2009, http://personales.unican.es/iglesias/CGGM2009/ 